\title{
Hypernuclei, dibaryon and antinuclei production in high energy heavy ion collisions: Thermal production vs. Coalescence ${ }^{\text {th }}$
}

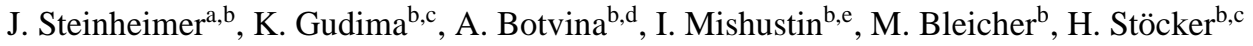 \\ ${ }^{a}$ Lawrence Berkeley National Laboratory, 1 Cyclotron Road, Berkeley, CA 94720, USA \\ ${ }^{b}$ FIAS, Johann Wolfgang Goethe University, Frankfurt am Main, Germany \\ ${ }^{c}$ Institute of Applied Physics, Academy of Sciences of Moldova, MD-2028 Kishinev, Moldova \\ ${ }^{d}$ Institute for Nuclear Research, Russian Academy of Sciences, 117312 Moscow, Russia \\ ${ }^{e}$ Kurchatov Institute, Russian Research Center, 123182 Moscow, Russia \\ ${ }^{f}$ GSI Helmholtzzentrum für Schwerionenforschung GmbH, Planckstr. 1, D-64291 Darmstadt, Germany
}

\begin{abstract}
We study the production of (hyper-)nuclei and di-baryons in most central heavy Ion collisions at energies of $E_{l a b}=1-160 \mathrm{~A} \mathrm{GeV}$. In particular we are interested in clusters produced from the hot and dense fireball. The formation rate of strange and non-strange clusters is estimated by assuming thermal production from the intermediate phase of the UrQMD-hydro hybrid model and alternatively by the coalescence mechanism from a hadronic cascade model. Both model types are compared in detail. For most energies we find that both approaches agree in their predictions for the yields of the clusters. Only for very low beam energies, and for di-baryons including $\Xi$ 's, we observe considerable differences. We also study the production of anti-matter clusters up to top RHIC energies and show that the observation of anti- ${ }^{4} \mathrm{He}$ and even anti- ${ }_{\Lambda}^{4} \mathrm{He}$ is feasible. We have found a considerable qualitative difference in the energy dependence of the strangeness population factor $R_{H}$ when comparing the thermal production with the coalescence results.
\end{abstract}

Keywords:

Hypernuclei, Dibaryons, Antinuclei, Relativistic Heavy Ion Collisions, Strangeness-Baryon Correlation

\section{Introduction}

Relativistic heavy ion collisions are an abundant source of strangeness. As strange quarks have to be newly produced during the hot and dense stage of the collision, they are thought of carrying information on the properties of the matter that was created [1]. Together with other probes like the elliptic flow and jet quenching, the enhancement of strange particle production is discussed $[2,3,4,5,6,6,7,8,9,10,11,12$, $13,14,15,16,17,18,19,20,21]$ as a possible signal for the creation of a deconfined phase.

Although abundantly produced, the strong interactions of strange hadrons are not well understood. Such interactions are not only important for the description of the hadronic phase of a heavy ion collision but also play an important role for the description of dense hadronic matter. In this context hyperon interactions are key to understand the phase structure of QCD at large densities and the interior of compact stars. One way to tackle the problem of hyperon interactions is to study the formation of hyperclusters and/or hypernuclei. Hypernuclear physics offers a direct experimental way to study hyperon-nucleon $(Y N)$ and hyperon-hyperon $(Y Y)$ interactions $(Y=\Lambda, \Sigma, \Xi, \Omega)$. The nucleus serves as a laboratory offering the unique opportunity to study basic properties of hyperons and their interactions. Even the confirmation or exclusion of the existence for such objects can be used as an input for models that try to describe hyperonic interactions.

More exotic forms of deeply bound objects with strangeness have been proposed [22] as states of matter, either consisting of baryons or quarks. The $\mathrm{H}$ di-baryon was predicted by Jaffe [23] and later, many more bound di-baryon states with strangeness were proposed using quark potentials [24, 25] or the Skyrme model [26]. However, the non-observation of multi-quark bags, e.g. strangelets is still one of the open problems of intermediate and high energy physics. Lattice calculations suggest that the H-dibaryon is a weakly unbound system [27], while recent lattice studies report that there could be strange di-baryon systems including 
$\Xi$ 's that can be bound [28]. Because of the size of these clusters lattice studies are usually very demanding on computational resources and have large lattice artifacts, it is not clear if Lattice QCD predicts a loosely bound H-dibaryon or if it is unbound [29, 30, 31, 32]. An experimental confirmation of such a state would therefore be an enormous advance in the understanding of the hyperon interaction.

For completeness we also include in our analysis a hypothetical $\mathrm{N} \Lambda$ di-baryon with mass $2.054 \mathrm{GeV}$ (see Table 1), a weakly bound state of a $\Lambda$-hyperon and a neutron. The search for such an exotic object is underway at GSI [33].

Hypernuclei are known to exist and be produced in heavy Ion collisions already for a long time [34, 35, 36, 37]. The recent discoveries of the first anti-hypertriton [38] and anti- $\alpha$ [39] (the largest antiparticle cluster ever reported) has fueled the interest in the field of hypernuclear physics. Metastable exotic multi-hypernuclear objects (MEMOs) as well as purely hyperonic systems of $\Lambda$ 's and $\Xi$ 's were introduced in [40, 41] as the hadronic counterparts to multi-strange quark bags [42, 43].

Hypernuclear clusters can be produced and studied in various experimental setups, e.g. from proton or antiproton induced reactions [44] as well as pion and kaon beams [45, 46, 47, 48, 49]. In this work we will focus on the production of hypernuclei in high energy collisions of $\mathrm{Au}+\mathrm{Au}$ ions [50]. In such systems strangeness is produced abundantly and is likely to form clusters of different sizes. Our aim is to determine which processes are most efficient in searching for hypernuclei including exotic ones. Presently, we can discriminate two distinct mechanisms for hypercluster formation in heavy ion collisions. First, the absorption of hyperons in the spectator fragments of non central heavy ion collisions. In this scenario one is interested in hyperons which propagate with velocities close to the initial velocities of the nuclei, i.e., in the vicinity of nuclear spectators [51, 52, 53, 54]. The hyper-systems obtained here are rather large and moderately excited, decaying into hyperfragments later on [54, 55]. Alternatively, (hyper)nuclear clusters can emerge from the hot and dense fireball region of the reaction. In this scenario the cluster is formed at, or shortly after, the (chemical-)freeze out of the system. A general assumption is, that these clusters are then formed through coalescence of different newly produced hadrons [56]. To estimate the production yield we can employ two distinct approaches which allow us to estimate the theoretical uncertainties associated with different treatment of the process. First

\begin{tabular}{|c|c|c|c|}
\hline Cluster & Mass [GeV] & Chem. Pot. & Spin Deg. \\
\hline \hline$d$ & 1.878 & $2 \mu_{B}$ & 3 \\
\hline$\{N \Lambda\}$ & 2.054 & $2 \mu_{B}-\mu_{S}$ & 3 \\
\hline$\{\Lambda \Lambda\}$ & 2.232 & $2 \mu_{B}-2 \mu_{S}$ & 1 \\
\hline$\{N \Xi\}$ & 2.260 & $2 \mu_{B}-2 \mu_{S}$ & 1 \\
\hline$\{\Lambda \Xi\}$ & 2.437 & $2 \mu_{B}-3 \mu_{S}$ & 1 \\
\hline$\{\Xi \Xi\}$ & 2.636 & $2 \mu_{B}-4 \mu_{S}$ & 1 \\
\hline$H e^{3}$ & 2.817 & $3 \mu_{B}$ & 2 \\
\hline$H e^{4}$ & 3.756 & $4 \mu_{B}$ & 1 \\
\hline${ }_{\Lambda}^{3} H$ & 2.994 & $3 \mu_{B}-\mu_{S}$ & 2 \\
\hline${ }_{\Lambda}^{4} H$ & 3.933 & $4 \mu_{B}-\mu_{S}$ & 1 \\
\hline${ }_{\Lambda}^{5} H e$ & 4.866 & $5 \mu_{B}-\mu_{S}$ & 2 \\
\hline${ }_{\Lambda \Lambda}^{4} H e$ & 4.110 & $4 \mu_{B}-2 \mu_{S}$ & 1 \\
\hline & & & \\
\hline
\end{tabular}

Table 1: Properties of all considered multibaryonic states

we use a hadronic transport model to provide us with the phase space information of all hadrons produced in a heavy ion collision. This information then serves as an input for a coalescence prescription. On the other hand it has been shown [57, 58, 59] that thermal models consistently describe the production yields of hadrons (and nuclei [60]) very well. We can therefore assume thermal production of clusters from a fluid dynamical description to heavy ion collisions.

Both approaches differ significantly in their assumptions and one would expect to obtain different results, depending on the method used. Hence it has been proposed (e.g. see [61, 62]) that the yield of an exotic hadronic state may depend strongly on its structure. The purpose of this paper is therefore to, comprehensively, compare hypernuclei and di-baryon production from a coalescence and thermal/hydrodynamical approach, and interpret the differences. One particular important point is that we deliberately compared two distinctively different models to explore the robustness of our predictions. In this way we can estimate systematic differences introduced by the two models features, for example a difference in the baryon stopping or hyperon phase space distributions.

\section{Thermal production from the UrQMD hybrid model}

The hybrid approach used in this work is based on the integration of a hydrodynamic evolution into the UrQMD transport model [63, 64, 65]. During the first phase of the evolution the particles are described by UrQMD as a string/hadronic cascade. Once the two colliding nuclei have passed through each other the hydrodynamic evolution starts at the time 
$t_{\text {start }}=2 R / \sqrt{\gamma_{c . m .}^{2}-1}$, where $\gamma_{c . m .}$ denotes the Lorentz factor of the colliding nuclei in their center of mass frame. While the spectators continue to propagate in the cascade, all other particles, i.e. their baryon charge densities and energy-momentum densities, are mapped to the hydrodynamic grid. By doing so one explicitly forces the system into a local thermal equilibrium for each cell. In the hydrodynamic part we solve the conservation equations for energy and momentum as well as the net baryon number current, while for the net strange number we assume it to be conserved and equal to zero locally. Solving only the equations for the net baryon number is commonly accepted in hydrodynamical models, although we have shown in earlier [66] publications that net strangeness may fluctuate locally. It is planned to also implement an explicit propagation for the net strange density.

The hydrodynamic evolution is performed using the SHASTA algorithm [67]. At the end of the hydrodynamic phase the fields are mapped to particle degrees of freedom using the Cooper-Frye equation [68] with the properties of the clusters, which serve as input for the computation, being listed in Table 1 The transition from the hydrodynamic prescription to the transport simulation is done gradually in transverse slices of thickness $0.2 \mathrm{fm}$, once all cells in a given slice have an energy density lower than five times the ground state energy density (see also [69]). The temperature at $\mu_{B}=0$ which corresponds to such a switching density is roughly $T=170 \mathrm{MeV}$ which is close to what is expected to be the critical temperature. Detailed information of the transition curve in the phase diagram can be found in [63]. In this work we neglected final state interactions of the clusters produced. This can be justified, as previous works have shown that final state interactions reduce e.g. the deuteron yield by only about $20 \%$ [70].

For an extensive description of the model the reader is referred to [63, 69].

\section{Coalescence from the DCM-QGSM approach}

Another model used to describe the dynamical stage of the reaction is the intra-nuclear cascade model developed in Dubna [71, 72]. (We refer to it as the Dubna Cascade Model - DCM.) The DCM is based on the Monte-Carlo solution of a set of the BoltzmannUehling-Uhlenbeck relativistic kinetic equations with the collision terms, including cascade-cascade interactions. For particle energies below $1 \mathrm{GeV}$ it is sufficient to consider only nucleons, pions and deltas. The

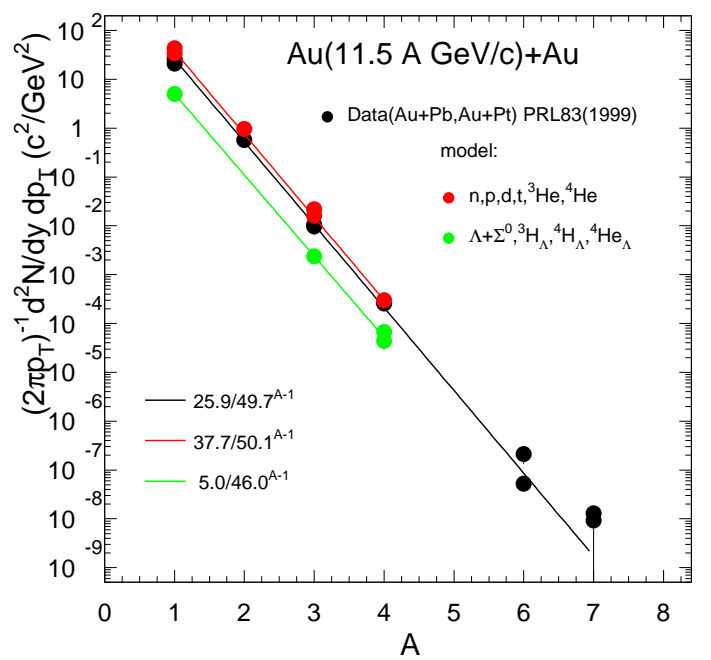

Figure 1: Mass dependence of calculated invariant yields of light fragments and hyperfragments produced in central $\mathrm{Au}+\mathrm{Au}$ collisions at 11.5 A GeV/c compared with experimental data 73 for $\mathrm{Au}+\mathrm{Pb}$ collisions. The lines are empirical interpolations of the results.

model includes a proper description of pion and baryon dynamics for particle production and absorption processes. At energies higher than about $10 \mathrm{GeV}$, the Quark-Gluon String Model (QGSM) is used to describe elementary hadron collisions. The QGSM considers the two lowest SU(3) multiplets in mesonic, baryonic and antibaryonic sectors, so interactions between almost 70 hadron species are treated on the same footing. The above noted two energy extremes were bridged by the QGSM extension downward in the beam energy [72].

For the present study the coalescence model has been modified in comparison with its initial formulation in [78]. As usual, the coalescence model forms a deuteron from a proton and a neutron produced after the cascade stage of reaction if their relative momenta are within a sphere of radius $p_{C}$, comparable to the deuteron's momentum. The same momentum criterion can be used to describe formation of tritons, ${ }^{3} \mathrm{He}$, and $\alpha$-particles. In particular, the parameters $p_{C}(d)=90, p_{C}(t)=108$, $p_{C}\left({ }^{3} \mathrm{He}\right)=108$, and $p_{C}(\alpha)=115(\mathrm{MeV} / \mathrm{c})$ were adopted to reproduce the experimental data [78]. An approach disregarding the spacial coordinates of nucleons can be justified only for collisions with moderate energy deposition in nuclei since the region for final state interaction is small enough. However, this is not the case for cen- 
tral heavy ion collisions. Here we assume that the coalescence criterion used to form the composite particles includes the proximity of nucleons both in the momentum and coordinate space. The coordinate coalescence parameters are determined by the relation $r_{C}=\hbar / p_{C}$, with the same values of $p_{C}$ as were used in [78]. As a first approximation we use the same coalescence parameters for both conventional fragments and hyperfragments. An example of the calculated invariant yields of the fragments produced in the central $\mathrm{Au}+\mathrm{Au}$ collisions at projectile momentum $11.5 \mathrm{~A} \mathrm{GeV}$ is shown in Fig. 1. One can understand that at this energy the coalescence model reproduces qualitatively the experimental data for conventional fragments. The fragments yields fit very close to exponential dependence with a penalty factor of approximately 50 for each nucleon added in agreement with the data. Due to the fact that the same coalescence parameters were used a similar penalty factor is obtained for hyperfragments, which is supplemented by additional suppression if the neutron is replaced by a $\Lambda$.

For the following results we fixed the coalescence parameters as described, with a fit to the data at $11.5 \mathrm{~A}$ $\mathrm{GeV}$, and assume that they do not change with beam energy. This allows us to predict cluster production over a wide range of experimental setups.

\section{Results}

Figures 2 and 3 show our results for the mid rapidity yields $(|y|<0.5)$ of di-baryons and hypernuclei as a function of the beam energy $E_{l a b}$. In our calculations we considered most central $(b<3.4 \mathrm{fm}) \mathrm{Pb}+\mathrm{Pb} / \mathrm{Au}+\mathrm{Au}$ collisions at $E_{l a b}=1-160 A \mathrm{GeV}$. In addition, figure 2 shows the $\Lambda$ yield (black lines and squares) for the two different models compared to data [75, 76, 77]. In these figures, the UrQMD hybrid model calculations are shown as lines, while the DCM Coalescence results are depicted as symbols. A striking feature of our comparison is that, above $E_{l a b} \sim 10 \mathrm{~A} \mathrm{GeV}$, both computations for most (hyper-)nuclei and di-baryons agree very well. At lower energies the strange cluster production is suppressed in the transport model due to the non-equilibrium of strangeness. In the thermal calculations restrictions of energy and momentum conservation, resulting in a phase space reduction for produced strange particles, strongly decreases strange particle yields [57, 58, 59]. This behavior was also observed in a core-corona implementation in the hybrid model [79].

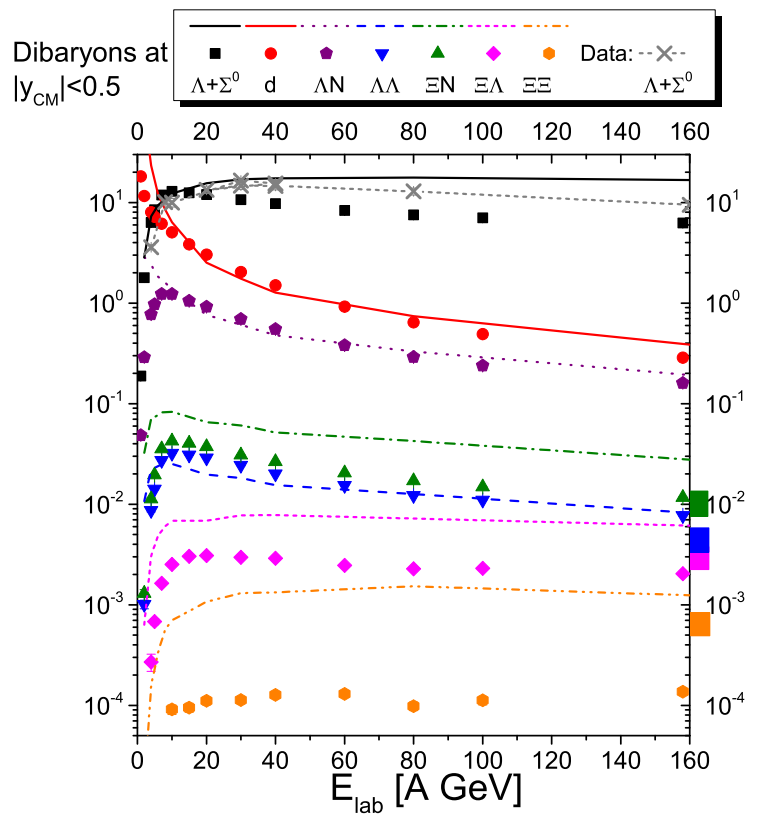

Figure 2: Yields per event of different di-baryons in the mid rapidity region $(|y|<0.5)$ of most central collisions of $\mathrm{Pb}+\mathrm{Pb} / \mathrm{Au}+\mathrm{Au}$. Shown are the results from the thermal production in the UrQMD hybrid model (lines) as compared to coalescence results with the DCM model (symbols). The small bars on the right hand axis denote results on dibaryon yields from a previous RQMD calculation at $\sqrt{s_{N N}}=200$ $\mathrm{GeV}[74]$. In addition, the black lines and symbols depict results for the production rate of $\Lambda$ 's from both models, compared to data (grey crosses) from [75, 76, 77].

An instructive result is that the yields of most hypernuclei have a maximum (or saturation) around 10-20 A GeV of beam energy. Therefore, the investigation of hypernuclei can be effectively pursued at these energies. On the other hand, the dependence of their yields up to energies of $\sim 200$ A GeV can help to clarify the mechanisms of hypernuclei production.

Noticeably the yields for di-baryons inlcuding $\Xi$ hyperons differ strongly with respect to the model applied, for the double $\Xi$ state the difference is as large as one order of magnitude. The reason for this discrepancy can be understood considering that the DCM model produces considerably, by a factor of 5 times, less $\Xi$ 's than the UrQMD hybrid model, therefore also the dibaryon formation is strongly suppressed (note that the experimental $\Xi$ yield is quite well reproduced by the UrQMD-hybrid model [80, 79]).

Di-baryon production rates have also been calculated in a coalescence approach using the RQMD model for $\sqrt{s_{N N}}=200 \mathrm{GeV}$ collisions of Au nuclei [74]. To re- 


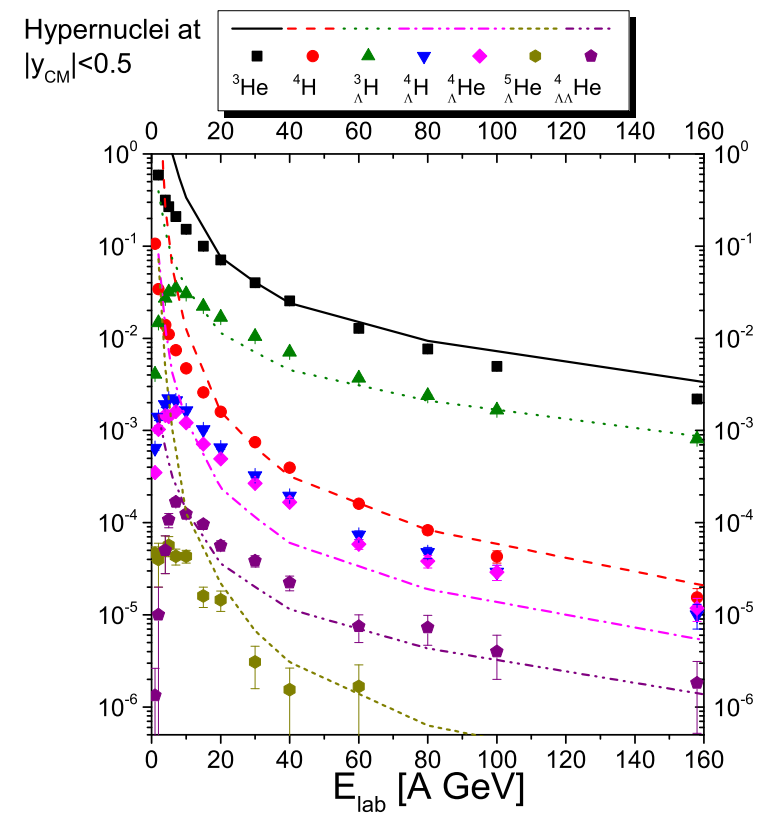

Figure 3: Yields per event of different (hyper-)nuclei in the mid rapidity region $(|y|<0.5)$ of most central collisions of $\mathrm{Pb}+\mathrm{Pb} / \mathrm{Au}+\mathrm{Au}$. Shown are the results from the thermal production in the UrQMD hybrid model (lines) as compared to coalescence results with the DCM model (symbols).

late our calculations to these results, they are indicated as the colored bars on the right axis of figure 2, The RQMD model used was in particular tuned to reproduce multi strange particle yields (such as the $\Xi$ ) and the results are therefore close to the ones obtained with our thermal/hydrodynamic approach.

Figures 4 and 5 show the integrated $(4 \pi)$ yields for all considered clusters as a function of beam energy. As with the midrapidity results there is a remarkable agreement between both approaches. However, the integrated yields of non-strange nuclei at high energies are systematically larger in the coalescence approach, although the mid-rapidity yield was smaller. This observation can be explained when the rapidity distribution of the nuclei is considered. In the coalescence approach the probability to produce a nucleus increases with rapidity and in particular in the fragmentation region, where the nucleons have small relative transverse momenta and can easily coalesce.

In addition we point out that the coalescence results depend on the parameters of the model. As mentioned, in the presented results the parameter $p_{C}$ for $\Lambda$ 's was taken equal to the one of the nucleon's. However, the hyperon-hyperon and hyperon-nucleon interactions are

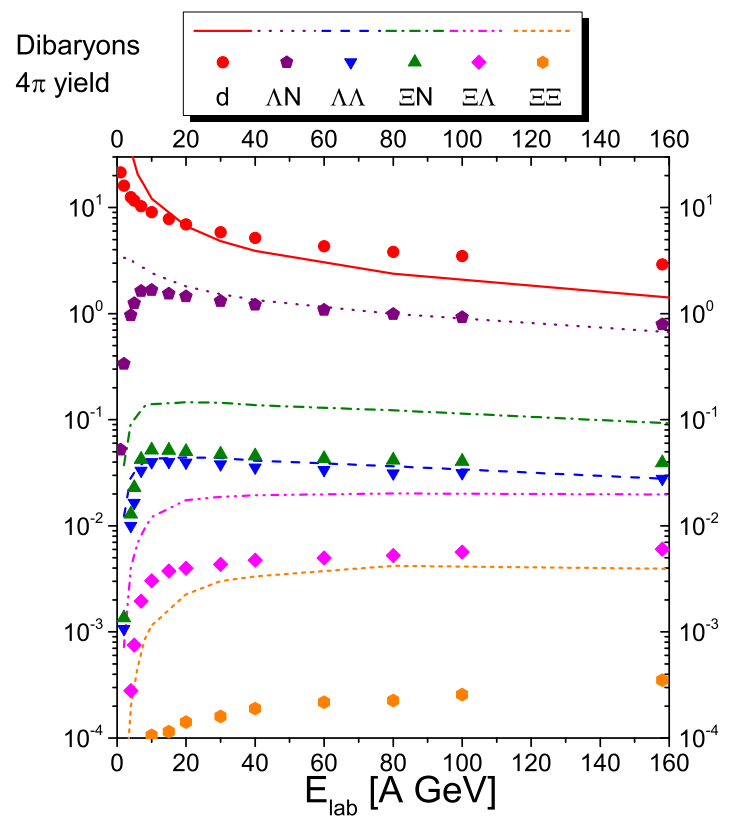

Figure 4: Full acceptance yields per event of different di-baryons created in most central collisions of $\mathrm{Pb}+\mathrm{Pb} / \mathrm{Au}+\mathrm{Au}$. Shown are the results from the thermal production in the UrQMD hybrid model (lines) as compared to coalescence results with the DCM model (symbols).

not very well known and we expect that these parameters may be different for clusters containing $\Lambda$ 's or even $\Xi$ 's. In table 2 we demonstrate how the yields of strange dibaryon nuclei depend on the momentum parameter $p_{C}$. As discussed previously, we have accordingly restricted the $r_{C}$ parameter, however, by imposing an empirical limitation related to the nuclear force properties that $r_{C}$ can not be larger than $4 \mathrm{fm}$. One can see, we expect a very large variation of the yields depending on the parameters. For instance, the probability of a bound $\Lambda$-nucleon state may decrease by many orders, if we assume a small $p_{C}$ corresponding to a low binding energy of this state. Usually the parameters are fixed by comparison with experiment. Nevertheless, ratios of hyper-

\begin{tabular}{|c|c|c|c|c|}
\hline$p_{C}=$ & 5 & 20 & 50 & 90 \\
\hline \hline$\Lambda \mathrm{N}$ & $4.4 \cdot 10^{-4}$ & $2.7 \cdot 10^{-2}$ & $3.0 \cdot 10^{-1}$ & 2.1 \\
\hline$\Lambda \Lambda$ & $3.0 \cdot 10^{-5}$ & $1.2 \cdot 10^{-3}$ & $6.6 \cdot 10^{-3}$ & $5.6 \cdot 10^{-2}$ \\
\hline$\Xi \mathrm{N}$ & $<10^{-6}$ & $1.0 \cdot 10^{-3}$ & $1.1 \cdot 10^{-2}$ & $1.0 \cdot 10^{-1}$ \\
\hline$\Xi \Lambda$ & $<10^{-6}$ & $7.4 \cdot 10^{-5}$ & $5.8 \cdot 10^{-4}$ & $1.0 \cdot 10^{-2}$ \\
\hline$\Xi \Xi$ & $<10^{-6}$ & $<10^{-6}$ & $3.8 \cdot 10^{-4}$ & $7.2 \cdot 10^{-4}$ \\
\hline
\end{tabular}

Table 2: Dependence of yield of strange dibaryons (per one event) on momentum coalescence parameter $\left(p_{C}\right.$ in units of $\left.[\mathrm{MeV} / \mathrm{c}]\right)$, in central $(b<3.5 \mathrm{fm}) \mathrm{Au}+\mathrm{Au}$ collisions at $20 \mathrm{~A} \mathrm{GeV}$ 


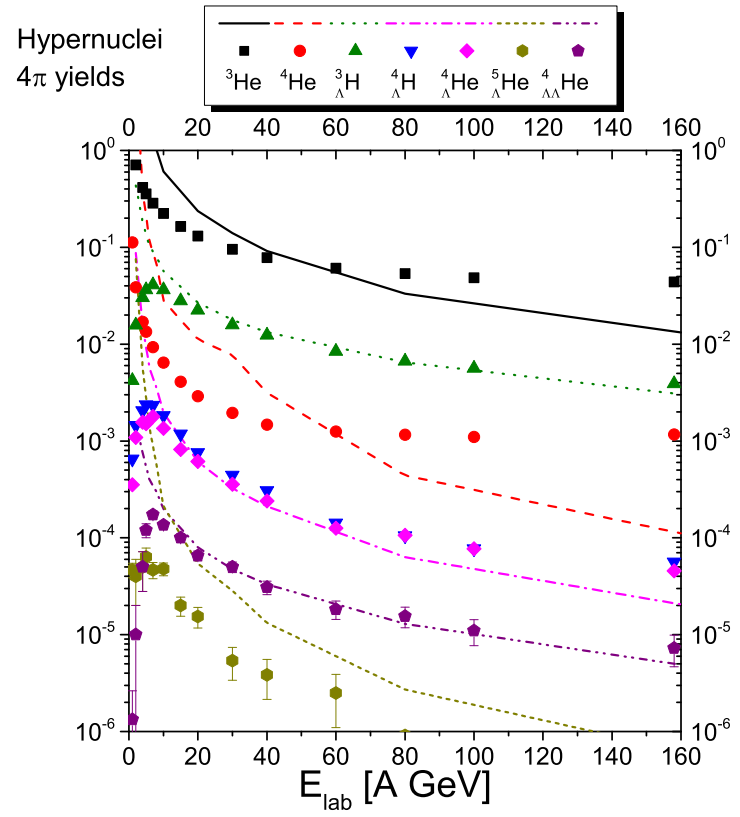

Figure 5: Full acceptance yields per event of different (hyper-)nuclei created in most central collisions of $\mathrm{Pb}+\mathrm{Pb} / \mathrm{Au}+\mathrm{Au}$. Shown are the results from the thermal production in the UrQMD hybrid model (lines) as compared to coalescence results with the DCM model (symbols).

nuclei yields should not be changed in the coalescence model.

When the beam energy of the collisions is increased, the system created becomes almost net-baryon free. This means that the probability to create an anti-particle cluster approaches that of the particle cluster. Figure 6 shows the results for anti-particle cluster production at mid-rapidity $(|y|<0.5)$ in collisions of $\mathrm{Pb}+\mathrm{Pb} / \mathrm{Au}+\mathrm{Au}$ at center of mass energies of $\sqrt{s_{N N}}=3-200 \mathrm{GeV}$. We show only results for the UrQMD hybrid model because the DCM calculations are restricted to energies up to $E_{l a b}=160 \mathrm{~A} \mathrm{GeV}$ where the statistics needed for a meaningful estimate are quite significant. The yields of the anti-particle clusters show a monotonous increase with beam energy. They show that, at the highest RHIC energy (and at the LHC) the reconstruction of ${ }_{\Lambda}^{4} \mathrm{He}$ might be a feasible task.

\subsection{A special ratio}

In the following we will discuss the double ratio $R_{H}$ defined as:

$$
R_{H}={ }_{\Lambda}^{3} H /{ }^{3} H e \cdot p / \Lambda
$$

for collisions of $\mathrm{Pb}+\mathrm{Pb} / \mathrm{Au}+\mathrm{Au}$ and a wide range of beam energies. This ratio is especially interesting, as in

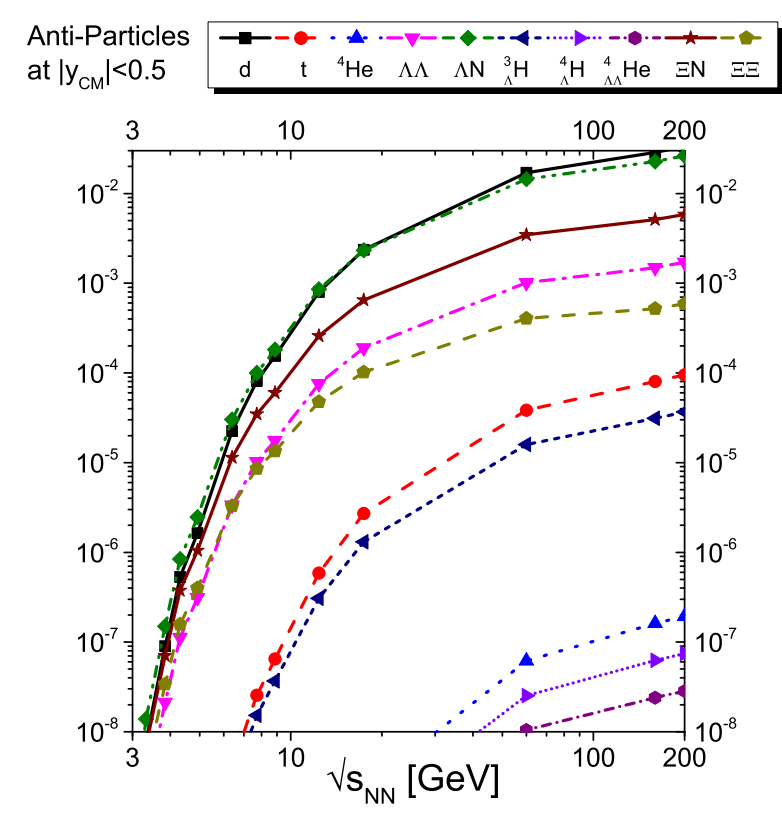

Figure 6: Yields of anti-particle clusters in the mid rapidity region $(|y|<0.5)$ of most central collisions of $\mathrm{Pb}+\mathrm{Pb} / \mathrm{Au}+\mathrm{Au}$ as a function of $\sqrt{s_{N N}}$. Shown are only the results from the thermal production in the UrQMD hybrid model (lines with symbols).

thermal production, it does not depend on the chemical potential of the particles (as fugacities cancel), and any canonical correction factors for strangeness are canceled. It has been proposed that this ratio is sensitive to the local correlation of strangeness and baryon number, therefore being a measure of $c_{B S}$ [81].

$$
c_{B S}=-3 \frac{\left\langle N_{B} N_{S}\right\rangle-\left\langle N_{B}\right\rangle\left\langle N_{S}\right\rangle}{\left\langle N_{S}^{2}\right\rangle-\left\langle N_{S}\right\rangle^{2}}
$$

To calculate $R_{H}$ we use the above obtained yields for hypernuclei and the proton and $\Lambda$ yields from the same model. For the hadrons the feed down from resonances is taken into account as well as the feed down to the ${ }^{3} \mathrm{He}$ from the hypertriton.

Our results for $R_{H}$ are shown in figure 7 as an excitation function of the beam energy $\sqrt{s_{N N}} . R_{H}$ is evaluated for the mid rapidity region of most central $(b<3.4 \mathrm{fm})$ heavy ion collisions. The lines depict results from the UrQMD-hybrid model and the symbols denote DCM coalescence results. Experimental data are depicted as green symbols with error bars. Because experiments usually cannot distinguish between $\Lambda$ 's and $\Sigma^{0}$ 's, we show $R_{H}$ in the cases where the $\Lambda$ yield includes $\Sigma^{0}$ 


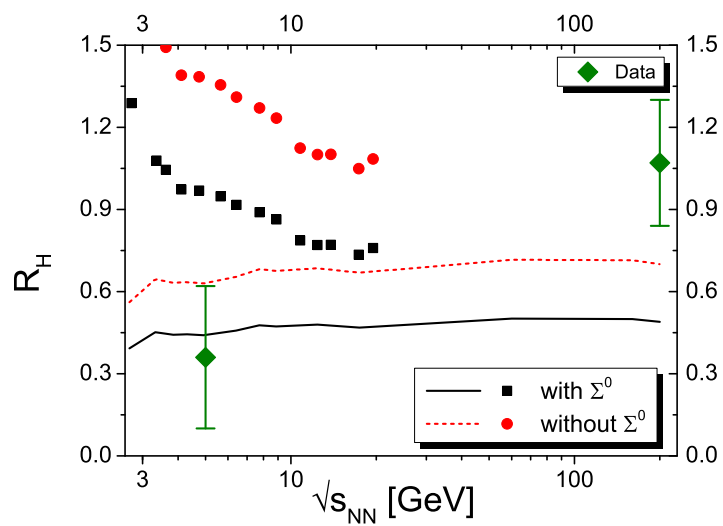

Figure 7: The Strangeness Population Factor $R_{H}=\left({ }_{\Lambda}^{3} H /{ }^{3} H e\right) \cdot(p / \Lambda)$ as a function of $\sqrt{s_{N N}}$ for most central collisions of $\mathrm{Pb}+\mathrm{Pb} / \mathrm{Au}+\mathrm{Au}$. We compare results from the thermal production in the UrQMD hybrid model (lines) with coalescence results with the DCM model (symbols). The red line and symbols denote values of $R_{H}$ where the $\Lambda$ yield has been corrected for the $\Sigma^{0}$ contribution.

(black solid line and squares) and where the yield is corrected for the $\Sigma^{0}$ (red dashed line and circles). This is in fact important as there is no experimental indication for a bound ${ }_{\Sigma^{0}}^{3} H$ hypernucleus.

The double ratio $R_{H}$ from the hybrid model turns out to be almost energy independent. The same behavior has been observed in previous thermal calculations [82]. On the other hand, the coalescence result increases with decreasing beam energy and is in general larger than the thermal result.

To understand this behavior we plotted the single ratios ${ }_{\Lambda}^{3} \mathrm{H} /{ }^{3} \mathrm{He}$ and $\Lambda / p$ from our two approaches (lines hybrid model and symbols DCM coalescence) in figure 8. Here it is obvious that even though the DCM calculation produces less $\Lambda$ 's per proton, the hypernuclei to nuclei ratio is still larger. Hence, the $\Lambda$ is more likely to form a hypernucleus. There seems to be a stronger correlation in the transport calculation as in the hydrodynamic description. In fact the qualitative behavior of $R_{H}$ closely resembles the behavior that is expected for $c_{B S}$, the baryon-strangeness correlation, for a hadronic gas [83]. This observation leads to the conclusion that the information on correlations of baryon number and strangeness is lost in the thermal calculation because here $R_{H}$ essentially only depends on the temperature. On the other hand, in the microscopic treatment the correlation information survives and $R_{H}$ captures the trend of $c_{B S}$.

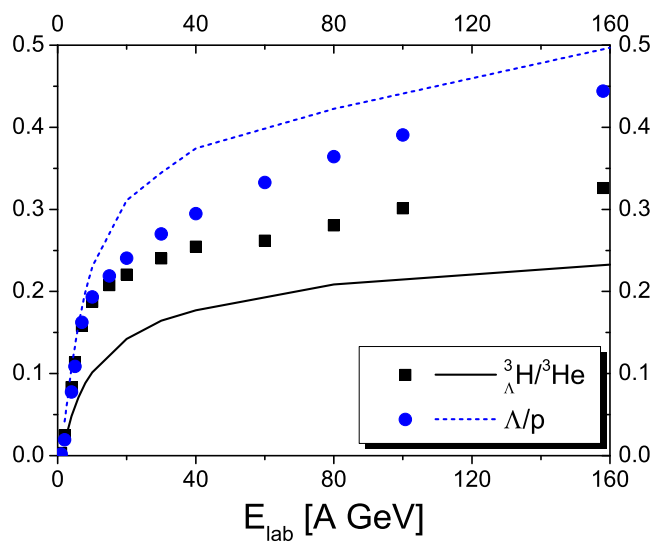

Figure 8: Single ratios of $\left({ }_{\Lambda}^{3} \mathrm{H} /{ }^{3} \mathrm{He}\right)$ (black solid line and circles) and $\Lambda / p$ (blue dashed line and squares) from the UrQMD hybrid model (lines) and DCM model (symbols).

\section{Conclusion}

We have presented results on hyper-nuclei, antinuclei and di-baryon production in heavy ion collisions over a wide beam energy range. To explore the theoretical uncertainties we applied two distinct approaches: firstly, the thermal production with the UrQMD-hydro hybrid model and secondly, the coalescence calculation within the Dubna hadron cascade model. Concerning most hyper-nuclei and di-baryons both approaches agree well in their predictions which gives us confidence in robustness and significance of the obtained results. We find that both the non-equilibrium and thermal models may be considered as appropriate approaches to describe strange cluster production. In agreement with previous studies we demonstrate that the most promising energy range to produce hyper-clusters will be provided by the FAIR and NICA facilities, $E_{l a b} \approx 10-20 \mathrm{~A} \mathrm{GeV}$. Anti-matter clusters heavier than $\bar{t}$ are only feasible at RHIC and LHC energies.

The most interesting result of our study is the apparent difference in the double ratio $R_{H}$ when we compare our thermal results with the coalescence. This difference indicates that the information on correlations of baryon number and strangeness are visible in the microscopic coalescence approach, while they are washed out in the thermal picture. This could open the opportunity to directly measure the strangeness-baryon correlation, which may be sensitive to the onset of deconfinement. The present status of the experimental data does unfor- 
tunately not allow for a comprehensive comparison with our model calculations. We hope that this situation will improve in the upcoming RHIC energy scan and FAIR experiments.

\section{Acknowledgments}

This work has been supported by GSI and Hessian initiative for excellence (LOEWE) through the Helmholtz International Center for FAIR (HIC for FAIR). J. S. acknowledges a Feodor Lynen fellowship of the Alexander von Humboldt foundation. This work was supported by the Office of Nuclear Physics in the US Department of Energy's Office of Science under Contract No. DE-AC02-05CH11231. I.M. acknowledges partial support from grant NS-215.2012.2 (Russia). The computational resources were provided by the LOEWE Frankfurt Center for Scientific Computing (LOEWE-CSC).

\section{References}

[1] P. Koch, B. Muller and J. Rafelski, Phys. Rept. 142, 167 (1986).

[2] J. Adams et al. [STAR Collaboration], Nucl. Phys. A 757, 102 (2005)

[3] B. B. Back et al., Nucl. Phys. A 757, 28 (2005)

[4] I. Arsene et al. [BRAHMS Collaboration], Nucl. Phys. A 757, 1 (2005)

[5] K. Adcox et al. [PHENIX Collaboration], Nucl. Phys. A 757, 184 (2005)

[6] J. Y. Ollitrault, Phys. Rev. D 46, 229 (1992).

[7] D. H. Rischke, Nucl. Phys. A 610, 88C (1996)

[8] H. Sorge, Phys. Rev. Lett. 78, 2309 (1997)

[9] H. Heiselberg and A. M. Levy, Phys. Rev. C 59, 2716 (1999)

[10] S. Scherer et al., Prog. Part. Nucl. Phys. 42, 279 (1999).

[11] S. Soff, S. A. Bass, M. Bleicher, H. Stoecker and W. Greiner, arXiv:nucl-th/9903061

[12] J. Brachmann et al., Phys. Rev. C 61, 024909 (2000)

[13] L. P. Csernai and D. Rohrich, Phys. Lett. B 458, 454 (1999)

[14] B. Zhang, M. Gyulassy and C. M. Ko, Phys. Lett. B 455, 45 (1999)

[15] P. F. Kolb, J. Sollfrank and U. W. Heinz, Phys. Rev. C 62, 054909 (2000)

[16] M. Bleicher and H. Stoecker, Phys. Lett. B 526, 309 (2002)

[17] H. Stoecker, Nucl. Phys. A 750, 121 (2005)

[18] X. 1. Zhu, M. Bleicher and H. Stoecker, Phys. Rev. C 72, 064911 (2005)

[19] H. Petersen, Q. Li, X. Zhu and M. Bleicher, Phys. Rev. C 74, 064908 (2006)

[20] M. Gazdzicki et al. [NA49 Collaboration], J. Phys. G 30, S701 (2004)

[21] M. Gazdzicki and M. I. Gorenstein, Acta Phys. Polon. B 30, 2705 (1999)

[22] A. R. Bodmer, Phys. Rev. D 4, 1601 (1971).

[23] R. L. Jaffe, Phys. Rev. Lett. 38, 195 (1977)

[24] J. T. Goldman, K. Maltman, G. J. . Stephenson, K. E. Schmidt and F. Wang, Phys. Rev. Lett. 59, 627 (1987).

[25] J. T. Goldman, K. Maltman, G. J. . Stephenson, J. L. . Ping and F. Wang, Mod. Phys. Lett. A 13, 59 (1998)
[26] B. Schwesinger, F. G. Scholtz and H. B. Geyer, Phys. Rev. D 51, 1228 (1995)

[27] I. Wetzorke and F. Karsch, Nucl. Phys. Proc. Suppl. 119, 278 (2003)

[28] S. R. Beane et al. [NPLQCD Collaboration and NPLQCD Collaboration and NPLQCD Collaboration], arXiv:1109.2889 [heplat].

[29] S. R. Beane et al. [NPLQCD Collaboration], Phys. Rev. Lett. 106, 162001 (2011)

[30] S. R. Beane, E. Chang, W. Detmold, B. Joo, H. W. Lin, T. C. Luu, K. Orginos and A. Parreno et al., Mod. Phys. Lett. A 26, 2587 (2011)

[31] T. Inoue et al. [HAL QCD Collaboration], Phys. Rev. Lett. 106, 162002 (2011)

[32] M. I. Buchoff, T. C. Luu and J. Wasem, arXiv:1201.3596 [heplat].

[33] T. Saito et al. [HypHi collaboration] invited talk presented at the NUFRA2011 conference.

[34] P. Braun-Munzinger and J. Stachel, J. Phys. G 21, L17 (1995)

[35] J. K. Ahn et al., Phys. Rev. Lett. 87, 132504 (2001).

[36] H. Takahashi et al., Phys. Rev. Lett. 87, 212502 (2001).

[37] A. Andronic, P. Braun-Munzinger, J. Stachel and H. Stocker, Phys. Lett. B 697, 203 (2011)

[38] STAR Collaboration, Science 328, (2010) 58

[39] STAR Collaboration, Nature 473 (2011) 353.

[40] J. Schaffner, H. Stoecker and C. Greiner, Phys. Rev. C 46, 322 (1992).

[41] J. Schaffner, C. B. Dover, A. Gal, C. Greiner and H. Stoecker, Phys. Rev. Lett. 71, 1328 (1993).

[42] E. P. Gilson and R. L. Jaffe, Phys. Rev. Lett. 71, 332 (1993)

[43] J. Schaffner-Bielich, C. Greiner, A. Diener and H. Stoecker, Phys. Rev. C 55, 3038 (1997)

[44] T. Gaitanos, A. B. Larionov, H. Lenske and U. Mosel, arXiv:1111.5748 [nucl-th]

[45] M. A. Faessler et al. [CERN-Heidelberg-Warsaw Collaboration], Phys. Lett. B 46, 468 (1973).

[46] R. E. Chrien et al., Phys. Lett. B 89, 31 (1979).

[47] M. Akei et al., Nucl. Phys. A 534, 478 (1991).

[48] F. Dohrmann et al. [Jefferson Lab E91-016 Collaboration], Phys. Rev. Lett. 93, 242501 (2004)

[49] O. Hashimoto and H. Tamura, Prog. Part. Nucl. Phys. 57, 564 (2006).

[50] A. J. Baltz, C. B. Dover, S. H. Kahana, Y. Pang, T. J. Schlagel and E. Schnedermann, Phys. Lett. B 325, 7 (1994).

[51] C. M. Ko, Phys. Rev. C 32, 326 (1985).

[52] T. Gaitanos, H. Lenske and U. Mosel, Phys. Lett. B 663, 197 (2008)

[53] T. Gaitanos, H. Lenske and U. Mosel, Phys. Lett. B 675, 297 (2009)

[54] A. S. Botvina, K. K. Gudima, J. Steinheimer, M. Bleicher and I. N. Mishustin, Phys. Rev. C 84, 064904 (2011)

[55] A. S. Botvina and J. Pochodzalla, Phys. Rev. C 76, 024909 (2007)

[56] R. Scheibl and U. W. Heinz, Phys. Rev. C 59, 1585 (1999)

[57] F. Becattini and U. W. Heinz, Z. Phys. C 76, 269 (1997) [Erratum-ibid. C 76, 578 (1997)]

[58] J. Cleymans, K. Redlich and E. Suhonen, Z. Phys. C 51, 137 (1991).

[59] A. Andronic, P. Braun-Munzinger and J. Stachel, Nucl. Phys. A 772, 167 (2006)

[60] A. Andronic, P. Braun-Munzinger and J. Stachel, Phys. Lett. B 673, 142 (2009) [Erratum-ibid. B 678, 516 (2009)]

[61] S. Cho et al. [ExHIC Collaboration], Phys. Rev. Lett. 106, 212001 (2011)

[62] S. Cho et al. [ExHIC Collaboration], Phys. Rev. C 84, 064910 
(2011)

[63] H. Petersen, J. Steinheimer, G. Burau, M. Bleicher and H. Stocker, Phys. Rev. C 78, 044901 (2008)

[64] H. Petersen, M. Bleicher, S. A. Bass and H. Stocker, arXiv:0805.0567 [hep-ph].

[65] J. Steinheimer, M. Bleicher, H. Petersen, S. Schramm, H. Stocker and D. Zschiesche, Phys. Rev. C 77, 034901 (2008)

[66] J. Steinheimer, M. Mitrovski, T. Schuster, H. Petersen, M. Bleicher and H. Stoecker, Phys. Lett. B 676, 126 (2009)

[67] D. H. Rischke, S. Bernard and J. A. Maruhn, Nucl. Phys. A 595, 346 (1995)

[68] F. Cooper and G. Frye, Phys. Rev. D 10, 186 (1974).

[69] J. Steinheimer, V. Dexheimer, H. Petersen, M. Bleicher, S. Schramm and H. Stoecker, Phys. Rev. C 81, 044913 (2010)

[70] Y. Oh, Z. -W. Lin and C. M. Ko, Phys. Rev. C 80, 064902 (2009)

[71] V. D. Toneev, N. S. Amelin, K. K. Gudima and S. Y. Sivoklokov, Nucl. Phys. A 519, 463C (1990).

[72] N. S. Amelin, K. K. Gudima, S. Y. Sivoklokov and V. D. Toneev, Sov. J. Nucl. Phys. 52, 172 (1990) [Yad. Fiz. 52, 272 (1990)].

[73] T. A. Armstrong et al. [E864 Collaboration], Phys. Rev. C 61, 064908 (2000)

[74] J. Schaffner-Bielich, R. Mattiello and H. Sorge, Phys. Rev. Lett. 84, 4305 (2000)

[75] S. Ahmad et al., Phys. Lett. B 382, 35 (1996).

[76] A. Mischke et al. [NA49 Collaboration], J. Phys. G 28, 1761 (2002)

[77] C. Alt et al. [NA49 Collaboration], Phys. Rev. C 78, 034918 (2008)

[78] V. D. Toneev and K. K. Gudima, Nucl. Phys. A 400, 173C (1983).

[79] J. Steinheimer and M. Bleicher, Phys. Rev. C 84, 024905 (2011)

[80] J. Steinheimer, H. Petersen, G. Burau, M. Bleicher and H. Stoecker, Acta Phys. Polon. B 40, 999 (2009).

[81] S. Zhang, J. H. Chen, H. Crawford, D. Keane, Y. G. Ma and Z. B. Xu, Phys. Lett. B 684, 224 (2010)

[82] A. Andronic, P. Braun-Munzinger, J. Stachel and H. Stocker, Phys. Lett. B 697, 203 (2011)

[83] V. Koch, A. Majumder and J. Randrup, Phys. Rev. Lett. 95, 182301 (2005) 